\title{
Role of Resistin with Endothelium Dysfunction in STEMI and NSTEMI Patients and Its Correlation with Cardiac Markers Troponins
}

\author{
Shazia Rashid ${ }^{1, ~ *}$, Javed Anver Qureshi ${ }^{1}$, Rukhshan Khurshid ${ }^{2}$, Huma Ashraf ${ }^{3}$, Saima Rasheed $^{4}$, \\ Uzma Faryal ${ }^{5}$ \\ ${ }^{1}$ Institute of Molecular Biology and Biotechnology, University of Lahore, Lahore, Pakistan \\ ${ }^{2}$ Department of Biochemistry, Shalamar Medical and Dental College, Lahore, Pakistan \\ ${ }^{3}$ Department of Biochemistry, CMH Lahore Medical College and Institute of Dentistry, Lahore, Pakistan \\ ${ }^{4}$ Department of Biochemistry, Niazi Medical and Dental College, Sargodha, Pakistan \\ ${ }^{5}$ Department of Biochemistry, Women Medical College, Abbottabad, Pakistan
}

Email address:

shazia.rashid9@gmail.com (S. Rashid)

${ }^{*}$ Corresponding author

\section{To cite this article:}

Shazia Rashid, Javed Anver Qureshi, Rukhshan Khurshid, Huma Ashraf, Saima Rasheed, Uzma Faryal. Role of Resistin with Endothelium Dysfunction in STEMI and NSTEMI Patients and Its Correlation with Cardiac Markers Troponins. International Journal of Clinical and Experimental Medical Sciences. Vol. 7, No. 5, 2021, pp. 152-159. doi: 10.11648/j.ijcems.20210705.14

Received: August 3, 2021; Accepted: September 13, 2021; Published: October 28, 2021

\begin{abstract}
Acute coronary syndrome (ACS) is the leading cause of mortality worldwide comprised with ST-segment elevation myocardial infarction (STEMI) and non ST segment elevation myocardial infarction (NSTEMI). A cross sectional study for a period from March 2015 to June 2016 was designed to find out the role of resistin in endothelium dysfunction in STEMI and NSTEMI patients and its correlation with cardiac markers troponins. 100 consented patients with diagnosed STEMI and NSTEMI with age range 40 to 70 years were included in the study. 50 age matched healthy subjects were taken as controls. Base line blood tests fasting blood sugar, blood urea, serum creatinine, serum total cholesterol and serum triglyceride were estimated by auto analyzer using standard kits. Special tests including serum resistin, cardiac troponin T and cardiac troponin I were estimated by the technique of ELISA. Results were analyzed by SPSS 20 and found that the base line parameters except serum creatinine were significantly raised in patients as compare to controls. Level of resistin and cardiac biomarkers cardiac troponin T (cTnT) and cardiac troponin I (cTnI) were significantly raised in STEMI patients as compared to NSTEMI patients. A significant positive correlation of resistin with cardiac troponin T and I was observed in STEMI patients only. It is therefore concluded that increased level of resistin along with cardiac markers may be presented as a predictor of injury of myocardium and in turn indicate the severity of AMI in STEMI patients.
\end{abstract}

Keywords: STEMI, NSTEMI, Resistin, Cardiac Troponin T, Cardiac Troponin I

\section{Introduction}

Acute coronary syndrome (ACS) is the leading cause of mortality worldwide, particularly in the developing countries. ACS is a discrepancy in the demand and consumption of oxygen in myocardium tissues. In cases of STEMI, the discrepancy may be ruptures of plague of coronary artery resulting in the formation of thrombus and block the coronary artery. However, NSTEMI results from narrowing of coronary artery or micro-embolization of thrombus [1, 2].

Resistin is a $12 \mathrm{Kda}$ protein mainly secretes from macrophages and monocytes. It may be a link between metabolic signals mediated inflammation and problem of atherosclerosis [3]. It is also involved in vascular ailments. It is suggested that in atheromas resistin is secreted by 
macrophages and promote inflammation of the vasculature. Additionally it promotes proliferation of smooth muscle cell and angiogenesis [4]. Clinically, a link between increased level of resistin and coronary artery disease was also observed and it is suggested that levels of resistin is found to be stepwise increase, depending on the number of segments or stenotic vessels $[5,6]$.

It is thought that resistin binds to cell membrane receptor TLR4, activated the signaling pathways of cells especially MAP kinase pathway. This pathway in-turn promote the secretion of cytokines that may act as pro-inflammatory agents resulting in the dysfunction of endothelium including altered vaso-relaxation, increased the process of thrombosis, increase permeability of membrane and raised adhesion of cells and help in the development of atherosclerosis [5, 7].

The best biomarker for finding the injury of myocardium should have high values in cardiac tissue, with high sensitivity and specificity for the identification of damage of myocardium. These are measurable in blood of patients initially after the beginning of symptoms, like chest pain [8]. Cardiac troponins are proteins that regulate the calcium based interaction of actin \& myosin, and take part in relaxation and contraction of striated muscle. Cardiac troponins are good predictor of AMI as these are highly sensitive and specific as compared to other biomarkers. It is stated the levels of troponin secreted in blood and elevation with time is directly related with size of infarcted muscle, thrombosis and alteration of perfusion of tissue of myocardium [9]. Release of troponin in STEMI patients has expectable kinetics with initial levels reflect the duration of ischemia [10]. On the other hand NSTEMIs are distinct and associated with cardiac muscle injury resulting in the elevation of troponin without changes in ECG [11].

Increased values of troponin 1 are a predictor of poor prognosis. It is proposed that in the occurrence of AMI, an increase level of Tn I inhibit the complex of troponin-thin filaments, and block the interaction of actin-myosin with intracellular calcium and resulting in impairment of regulation of contractibility of myocardium [12].

An association between the level of troponin and resistin is observed in patients with STEMI. Study stated that combined effect of troponin I and resistin may be a reason of elevated left ventricular ejection fraction (LVEF). Study demonstrated that levels of resistin were associated with degree of infarction in patients with STEMI. It is suggested that high levels of resistin have predictive value for the incidence of systolic heart failure in patients with STEMI with better specificity, sensitivity, and precision than the levels of troponin I [13]. Increased values of troponin $\mathrm{T}$ after chest pain indicate myocardial infarction and significantly related heart failure and cardiovascular death [14]. However a study found no link between the levels of circulating adipokine resistin and elevation of troponin in peri-procedural myocardial injury [15].
Patients with high blood glucose are more prone to develop cardiac muscle impairment, increased secretions of biomarkers of myocardial necrosis, and severe coronary syndromes [16].

\section{Material and Methods}

A cross sectional study was designed to find out the role of resistin in endothelium dysfunction in STEMI and NSTEMI patients and its correlation with cardiac markers troponins.

The cross sectional study was carried out in 100 patients aged from 40 to 70 years presenting with AMI in Punjab Institute of Cardiology (PIC) Lahore. Patients with diabetes mellitus, renal disease or muscle dystrophy were excluded from the study. 50 age matched healthy subjects were taken as controls. Prior consent of all subjects was obtained. History and investigations were recorded in proformas. Of the patients, 56\% were diagnosed with STEMI and $44 \%$ with NSTEMI. The duration of study was March 2015 to June 2016. The study protocol was permitted by Ethical Review Committee, Department of Research, Training \& Postgraduate Medical Education, Punjab Institute of Cardiology, Lahore. Written informed consent was obtained before enrollment from all the participants. A comprehensive questionnaire documenting information on demographic data, medical and family history of coronary artery disease was also completed for each subject.

Base line blood tests fasting blood sugar, blood urea, serum creatinine, serum total cholesterol and serum triglyceride were estimated by Auto Analyzer using standard kits. Special tests including serum resistin, troponin $\mathrm{T}$ and troponin I were estimated by the technique of ELISA.

\section{Statistical Analysis}

Data was analyzed by SPSS 20. Variables were expressed as mean $\pm \mathrm{SD}$. Comparison of variables of patients and controls were carried out by student ' $t$ ' test. Correlation between resistin and troponins were done by using Pearson Correlation Coefficient. $\mathrm{P}$ value $<0.05$ is taken as significant.

Results of Table 1: Among 100 cases, $31 \%$ cases were with age $<50$ years and $69 \%$ cases were with age of $>50$ years. Gender based difference showed that $78 \%$ male and $22 \%$ were female. BMI bases difference showed that $2 \%$ were underweight, $38 \%$ were with normal weight, $54 \%$ were overweight and $6 \%$ were obese. About 54\% cases having active life style, and $46 \%$ having sedentary life style. It is observed that among 70 cases presented with MI, 56\% diagnosed as STEMI and $44 \%$ were diagnosed as NSTEMI. Among 50 control cases $70 \%$ were male and $30 \%$ female. Most of the control subject presented with normal weight followed overweight and obese. 
Table 1. Demographic characteristics of Patients and controls.

\begin{tabular}{lll}
\hline & Cases N (\%) & Controls N (\%) \\
\hline Age & & 34 \\
$\leq 50$ years & 31 & 66 \\
$>50$ years & 69 & 70 \\
Gender & 78 & 30 \\
Male & 22 & 2 \\
Female & & 54 \\
BMI & 2 & 36 \\
Underweight $(<18.5)$ & 38 & 8 \\
Normal weight $(18.5-24.9)$ & 54 & \\
Overweight $(25.0-29.9)$ & 6 & - \\
Obese $(>30.0)$ & & \\
Life style & 54 & \\
Active & 46 & - \\
Sedentary & & \\
Type of MI & 56 & \\
STEMI & 44 & \\
NSTEMI & & \\
\hline
\end{tabular}

Table 2. History profile of cases.

\begin{tabular}{lll}
\hline & Frequency & Percentage \\
\hline History of hypertension & & 64 \\
Positive & 64 & 36 \\
Negative & 36 & 48 \\
History of smoking & & 52 \\
Positive & 48 & SD \\
Negative & 52 & 4.7 \\
Duration of chest pain & mean & \\
\hline
\end{tabular}

Table 2 presented the history profile of cases. It is observed that $64 \%$ cases have a history of hypertension and $36 \%$ cases have no history of hypertension. Among 100 cases $48 \%$ were smokers and 52\% patients were non-smokers. Mean duration of chest pain in cases was 6.17 hours.

Table 3. Comparison of baseline investigations in cases and their controls.

\begin{tabular}{llll}
\hline Parameters & Cases mean \pm SD & Controls mean \pm SD & P-value \\
\hline Serum Triglycerids $(\mathrm{mg} / \mathrm{dl})$ & $161.32 \pm 30.4$ & $170.35 \pm 42.8$ & $0.04^{*}$ \\
Total serum Cholesterol $(\mathrm{mg} / \mathrm{dl})$ & $201.90 \pm 48.35$ & $163.42 \pm 44.40$ & $0.000^{*}$ \\
Fasting Blood Sugar $(\mathrm{mg} / \mathrm{dl})$ & $120.73 \pm 51.13$ & $90.46 \pm 18.90$ & $0.000^{*}$ \\
Blood Urea $(\mathrm{mg} / \mathrm{dl})$ & $39.27 \pm 16.07$ & $25.97 \pm 9.44$ & $0.000^{*}$ \\
Serum Creatinine $(\mathrm{mg} / \mathrm{dl})$ & $0.98 \pm 0.40$ & $0.92 \pm 0.52$ & 0.24 \\
\hline
\end{tabular}

Baseline investigations between cases and control are presented as table 3 . It is observed that the level of lipid profile including triglyceride and cholesterol are significantly increased in cases as compared to controls.
Levels of blood urea and blood glucose are significantly increased in cases as compared to controls. On the other hand serum creatinine is in-significantly increased in cases as compared to controls.

Table 4. Comparison of cardiac markers between STEMI and NSTEMI cases.

\begin{tabular}{llll}
\hline Cardiac markers & Male Cases with STEMI mean \pm SD & Male Cases with NSTEMI mean \pm SD & P-value \\
\hline Resistin $(\mathrm{ng} / \mathrm{ml})$ & $27.48 \pm 8.37$ & $23.31 \pm 6.13$ & $<0.05$ \\
Troponin-T $(\mathrm{ng} / \mathrm{ml})$ & $1591.27 \pm 1423.89$ & $293.97 \pm 188.43$ \\
Troponin-I $(\mathrm{ng} / \mathrm{ml})$ & $15.61 \pm 12.04$ & $2.29 \pm 1.91$ & $<0.001$ \\
\hline
\end{tabular}

Comparison of cardiac markers between STEMI and NSTEMI cases is tabulated as table 4. It is observed levels cardiac markers resistin, troponin $\mathrm{t}$ and troponin I was significantly increased in male cases with STEMI as compared to male cases with NSTEMI.

Table 5. Correlation of resistin with cTnT and cTnI in patients with STEMI.

\begin{tabular}{ll}
\hline Correlation & r-value \\
\hline Serum resistin with cTnT & $0.69^{*}$ \\
Serum adiponectin with cTnI & $0.63^{*}$ \\
\hline
\end{tabular}

$* \mathrm{P}<0.5$ is significant. 
Table 6. Correlation of resistin with cTnT and cTnI in patients with NSTEMI.

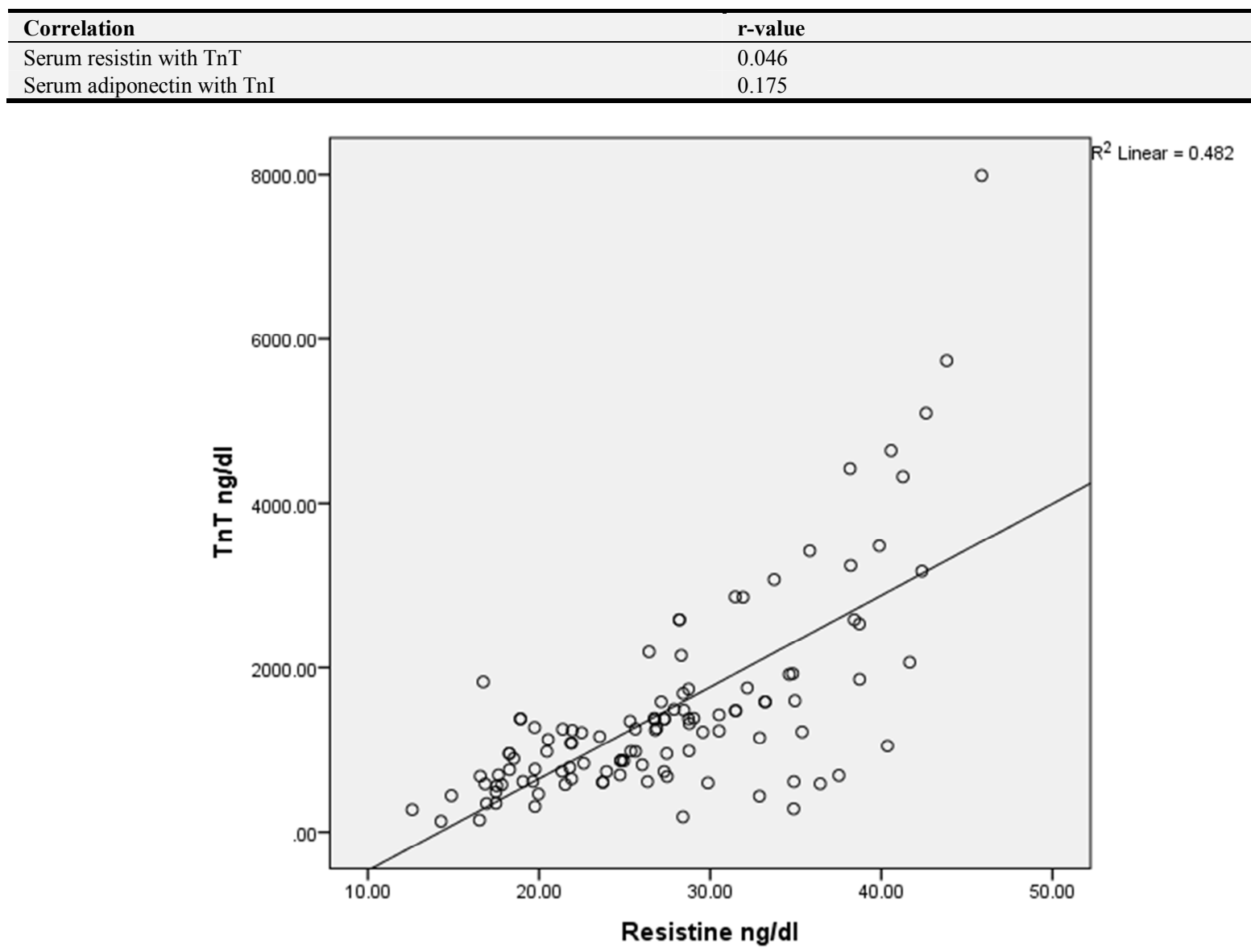

Figure 1. Significant positive correlation of resistin and cTnT in patients with STEMI.

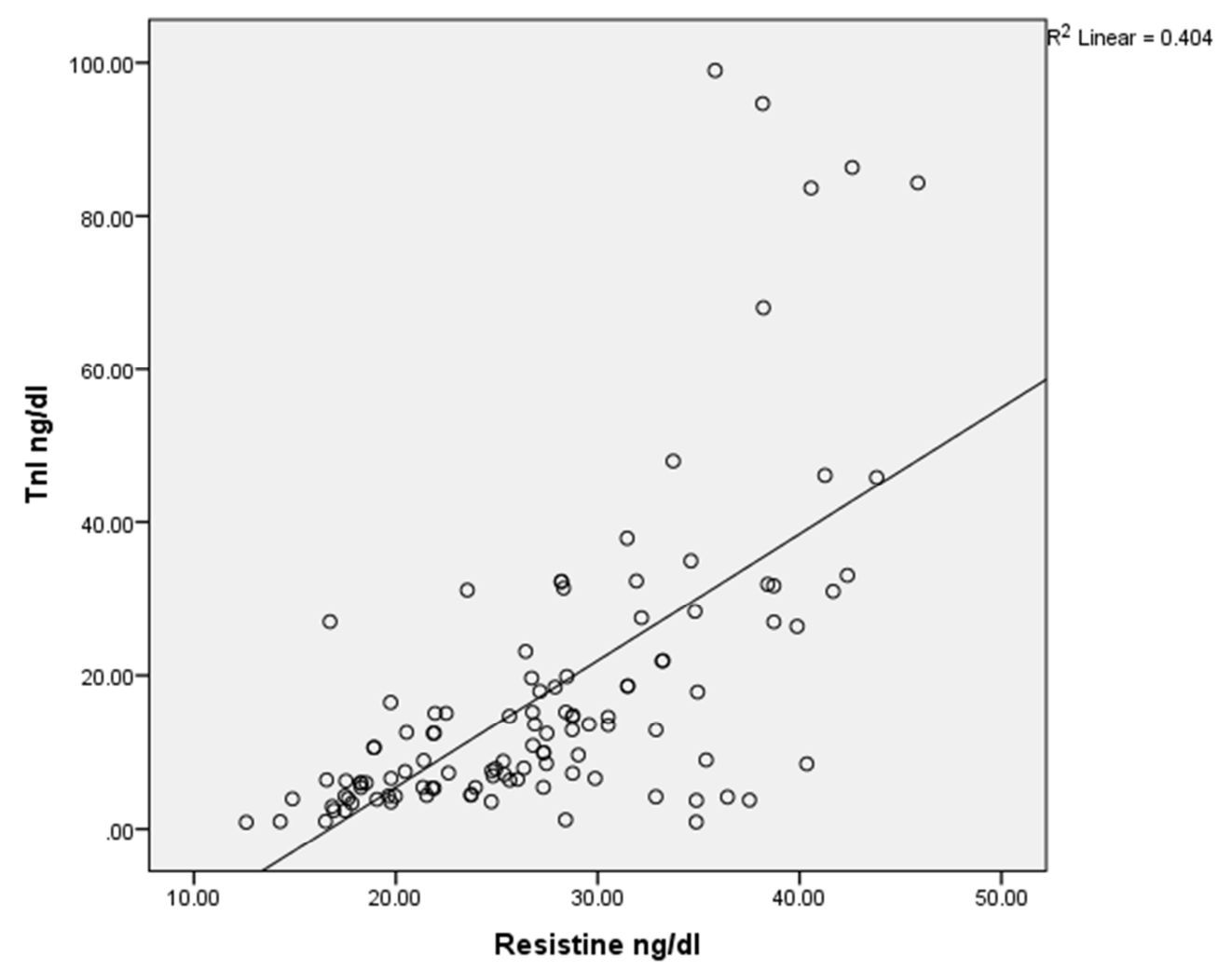

Figure 2. Significant positive correlation of resistin and cTnI in patients with STEMI. 


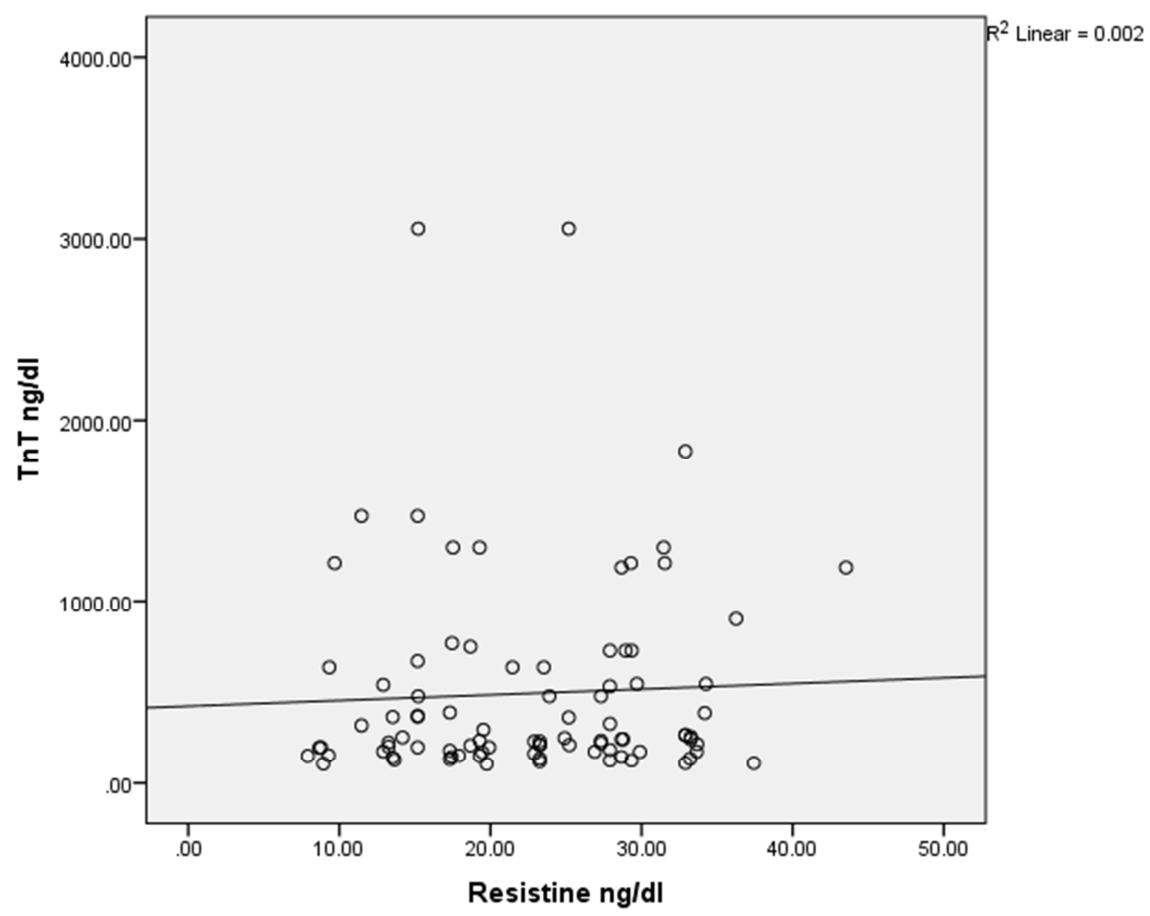

Figure 3. Positive correlation of resistin and cTnI in patients with NSTEMI.

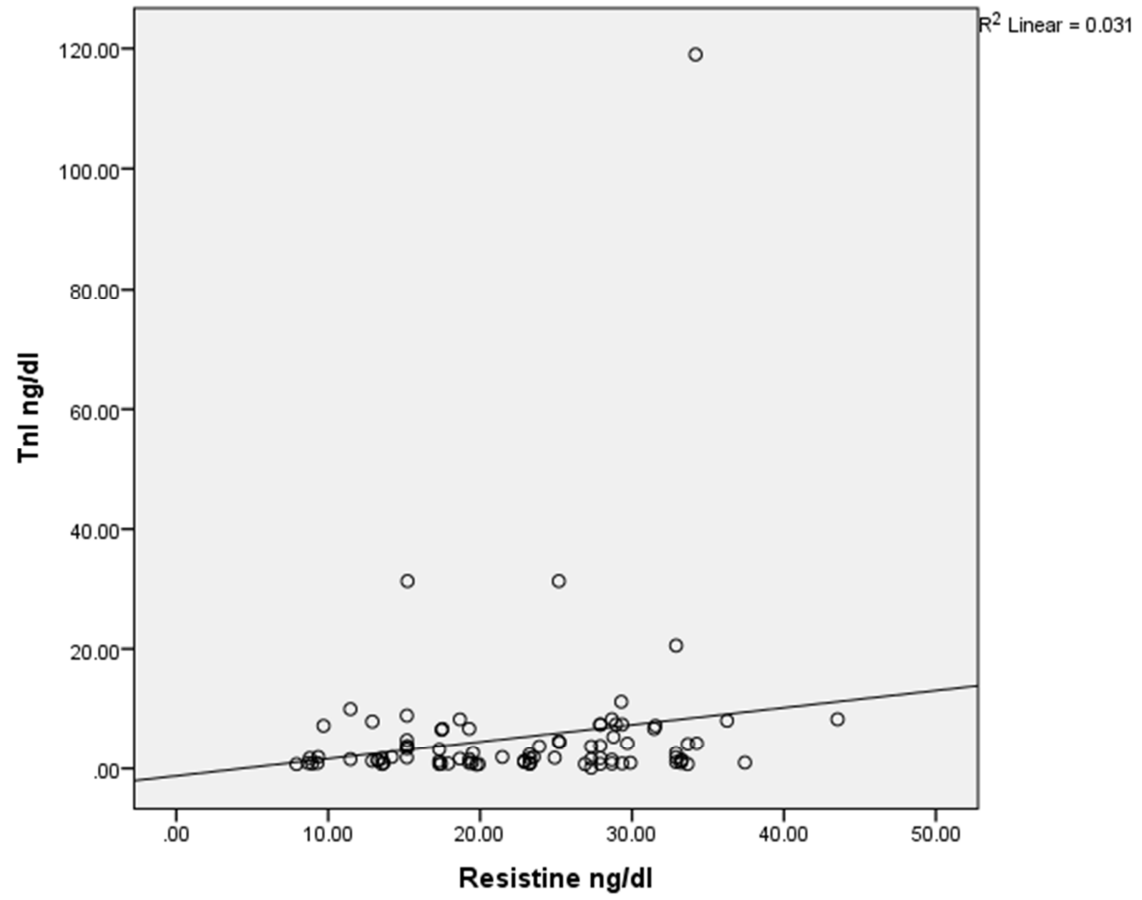

Figure 4. Positive correlation of resistin and cTnI in patients with NSTEMI.

Correlation studies showed that significant positive correlation $(\mathrm{P}<0.05)$ of resistin with $\mathrm{cTnT}$ and $\mathrm{cTnI}$ was observed in patients with STEMI. However positive insignificant correlation of resistin with cTnT and cTnI was observed in patients with NSTEMI.

\section{Discussion}

According to our results, AMI is more prevalent in male with age $>50$ years. Majority of patients are non-smoker but hypertensive. A study carried out on 484 Turkish male with first 3 to 6 hours of onset of symptom and found that AMI is more prevalent in age of more than 40 years (mean age 58 years) as compared to age less than 40 years onset were included in the study. [17].

Another study carried out on 100 ACS patients and revealed that hypertension is the furthermost known risk factor of developing ACS followed by type 2 diabetes and lastly smoking [18].

We observed that STEMI is more common in males as 
compared to NSTEMI. We agreed with a study carried out in US that STEMI in male has higher incidence than males with NSTEMI [19]. According to Brazilian Society of Cardiology morality rate due to STEMI is higher than NSTEMI [20]. However a study observed that majority of patients was smoker while small number of patients was hypertensive and NSTEMI is more common than STEMI [18].

Consistent with studies of old age cardiac patients, the base line investigation showed that lipid profile along with fasting blood sugar and blood urea were significantly raised in cases as compared to control except serum creatinine. A study carried out 100 patients with ACS and found dyslipidemia in both patients with STEMI and NSTEMI and stated that dyslipidemia is taken as a strong marker for outcomes of cardiovascular problems after AMI [17].

Another study was carried out on 200 patients with ACS and found that along with cardiac enzymes, the levels of blood urea /BUN and serum creatinine are high as compared to controls. Study concluded that blood urea/BUN and Creatinine may be significant additional tools in death-risk valuation of ACS patients and may provide detailed interpretation of the complications of ACS [21]. However in our study only blood urea was increased with no effect on the level of serum creatinine.

A total of 529 patients with the diagnosis of STEMI related acute coronary syndrome within $24 \mathrm{~h}$ of the onset of symptoms were included in the study. Study proposed two hypothesis. One is based that hyperglycemia in ACS patients is persuaded by activation of alpha /beta adrenergic receptors. Another one is based that it may be a predictor of preexisting, impaired metabolism of carbohydrate [22]. However it is reported that hyperglycemia is usually unnoticed in majority of AMI patients and the increase reason of mortality in ACS patients with less prognosis as compared to diabetics [23]. Another study suggested that hyperglycemia may be stress related in AMI and the level of blood glucose increased immediately after acute myocardial infarction regardless of diabetes status [23, 24].

We agreed with a study that observed a significantly high level of serum in patients with STEMI. Study also found no correlation of resistin with age, BMI, gender and fasting blood glucose. It is therefore suggested that level of serum resistin concentration may be a marker for diagnosis of STEMI [25]. Another study stated that high values of serum resistin may be presented as a predictor of injury of myocardium and inturn indicate the severity of AMI [6]. A study experimentally proved that high levels of resistin persuade resistance of leptin and increased the risk of progression of atherosclerosis [26].

It is proposed that resistin could stimulate endothelial cells and encourage the process of inflammation via chemokines and cytokines thus accelerating dysfunction of endothelium [27]. It also block the expression of endothelial nitric oxide encourage the production superoxide anion in endothelial cells, which decline the relaxation of endothelial-dependent vascular system and altered the process of relaxation and contraction in vessels of heart [28, 29].
A study was carried out on 100 hospitalized patients and found that $27 \%$ patients suffered with AMI and they have high values of serum resistin, which may be related with the severity of heart failure and at high risk of developing cardiac events signifying that high levels of serum resistin can estimate the forecasting of ailment and extent of heart problem and suggesting that reduction in the level of resistin may help in the treatment [6]. Additionally it is suggested that high levels of circulating resistin in patients with STEMI can be correlated with major hostile cardiac events [30]. In addition a cross sectional study on 92 patients with AMI also reported that resistin may control myocardial reperfusion injury by encouraging the process of inflammation and oxidative stress [31].

Correlation data showed that significant positive correlation of resistin was observed with cTnT and cTnI in STEMI as compared to NSTEMI patients. A study carried out AMI patients to find their levels of resistin and troponins and their correlation. Study reported that level of serum resistin is positively correlated with cardiac troponin I and suggested that values of serum resistin during the acute period of STEMI are valuable for predicting the size of myocardial infarction and prognosis in patients with ACS [32]. Another study also found the association of serum resistin with troponin I but reported that change in the level of serum resistin is not linked with injury of myocardium [26]. We agree with the study that was carried out on cardiac patients hospitalized in Finland. Study found that levels of resistin were increase significantly by 24 hour and positively correlated with release of troponin $\mathrm{T}$ [33]. However a study carried out on 153 old age patients and reported that resistin levels increased at 12.0 hours after the process but did not associate with increase values of troponins [15].

\section{Conclusion}

It is therefore concluded that increased level of resistin along with cardiac markers may be presented as a predictor of injury of myocardium and in turn indicate the severity of AMI in STEMI patients.

\section{Authors Contributions}

Dr Shazia Rashid --- Study Design, Data collection

Prof Dr Javed Anver Qureshi----Study Design

Dr Rukhshan Khurshid ---- Study Design, Paper Writing

Dr Huma Ashraf---- Literature Survey

Dr Saima Rasheed---Statistical Analysis

Prof Uzma Faryal----- Literature Survey and Proof reading

\section{References}

[1] Harrington DH, Stueben F, Lenahan CM. ST-Elevation Myocardial Infarction and Non-ST-Elevation Myocardial Infarction: Medical and Surgical Interventions. Crit Care NursClin North Am. 2019 Mar; 31 (1): 49-64. 
[2] Rupprecht HJ, Geeren M, Geeren M, Weilemann S, Schuster HP. [Acute coronary syndrome without ST-elevation (NSTEACS)]. Herz. 2019 Feb; 44 (1): 10-15.

[3] Tripathy D, Kant S, Pandey K, Ehtesham NZ. Resistin in metabolism, inflammation, and disease. The FEBS Journal 2020; 287: 3141-3149.

[4] Pang L, Zhang Y, Yu Y, Zhang S. Resistin promotes the expression of vascular endothelial growth factor in ovary carcinoma cells. Int J Mol Sci. 2013; 14 (5): 9751-9766. doi: 10.3390/ijms 14059751 .

[5] Jamaluddin MS, Weakley SM, Yao Q, Chen C. Resistin: functional roles and therapeutic considerations for cardiovascular disease. Br J Pharmacol. 2012; 165 (3): 622632. doi: 10.1111/j.1476-5381.2011.01369.x.

[6] Zhang J., Gao Y., Zheng Y., Liu F., Yang Y., Li X., Ma X., Ma Y., Xie X. Increased serum resistin level is associated with coronary heart disease. Oncotarget. 2017; 8: 50148-50154.

[7] Fang C, Lei J, Zhou SX, Zhang YL, Yuan GY, Wang JF. Association of higher resistin levels with inflammatory activation and endothelial dysfunction in patients with essential hypertension. Chin Med J (Engl). 2013 Feb; 126 (4): 646-9.

[8] R. P. Tiwari, A. Jain, Z. Khan et al., "Cardiac troponins I and T: molecular markers for early diagnosis, prognosis, and accurate triaging of patients with acute myocardial infarction," Molecular Diagnosis \& Therapy 2012; 16 (6): 371-381.

[9] Hajar R. Evolution of Myocardial Infarction and its Biomarkers: A Historical Perspective. Heart Views. 2016; 17 (4): 167-172. doi: 10.4103/1995-705X.201786.

[10] Wanamaker BL, Seth MM, Sukul D, Dixon SR, Bhatt DL, Madder RD et al. Relationship Between Troponin on Presentation and In-Hospital Mortality in Patients With STSegment-Elevation Myocardial Infarction Undergoing Primary Percutaneous Coronary Intervention. J Am Heart Ass. 2019; 8: e013551.

[11] Daubert MA, Jeremias A. The utility of troponin measurement to detect myocardial infarction: review of the current findings. Vasc Health Risk Manag. 2010; 6: 691-699. doi: $10.2147 /$ vhrm.s5306.

[12] Schoffstall B, LaBarbera VA, Brunet NM, et al. Interaction between troponin and myosin enhances contractile activity of myosin in cardiac muscle. DNA Cell Biol. 2011; 30 (9): 653659. doi: 10.1089/dna.2010.1163.

[13] Wasyanto T and Febrilia L. Association between Resistin and High Sensitive Troponin I in St Elevation Myocardial Infarction and Systolic Heart Failure. Indonesian J Med 2020; $5(1)$.

[14] Torbjørn O, De Lemos James A, Sabatine Marc S, Christophi Costas A, Murguia RM et al. "A Sensitive Cardiac Troponin T Assay in Stable Coronary Artery Disease" New Eng J Med 2009; 361 (26): 2538-2547.

[15] Buturak A, Değirmencioğlu A, Baryrak F, Kins T, Karakurt $\mathrm{H}$, Demir AR et al. Elective percutaneous coronary intervention leads to significant changes in serum resistin, leptin, and adiponectin levels regardless of periprocedural myocardial injury: an observational study. Anatol J Cardiol. 2016; $16 \quad$ (12): 940-946 | DOI: 10.14744/AnatolJCardiol.2016.687.
[16] Sasso FC, Rinaldi L, Lascar N, Marrone A, Pafundi PC, Adinolfi LE. Role of Tight Glycemic Control during Acute Coronary Syndrome on CV Outcome in Type 2 Diabetes. J Dia Res 2018; 2018: 8 pages https://doi.org/10.1155/2018/3106056

[17] Nilgun I, Ismail B, Caglar S, Fatma NT, Mehmet E, Serkan Y et al. Effect of age and gender difference on high sensitive troponin $\mathrm{T}$ measurement in the diagnosis of myocardial infarction. J Lab Med 2019; 43 (1): $35-40$ doi.org/10.1515/labmed-2018-0326.

[18] Patange A, Immadisetty S, Vora H, et al. Lipid levels in acute coronary syndromes admitted in medical intensive care unit in a tertiary care hospital. J. Evolution Med. Dent. Sci. 2020; 9 (08): 520-523, DOI: 10.14260/jemds/2020/117.

[19] Lichtman JH, Leifheit EC, Safdar B, Bao H, Krumholz HM, Lorenze NP et al. Sex Differences in the Presentation and Perception of Symptoms Among Young Patients With Myocardial Infarction. Circulation. 2018; 137: 781-790. DOI: 10.1161/CIRCULATIONAHA.117.031650.

[20] Junior Á, Feldman A, Carvalho AC, Sousa AC, Mansur Ade P, Bozza AE et al. Guideline of the Brazilian Society of Cardiology on Acute Myocardial Infarction Treatment with ST Segment Elevation]. Arq Bras Cardiol. 2015 Aug; 105 (2 Suppl 1): 1-105. Portuguese. doi: 10.5935/abc.20150107.

[21] Adam AM, Nasir SAR, Merchant AZ, Rizvi AH, Rehan A, Shaikh ATet al. Efficacy of serum blood urea nitrogen, creatinine and electrolytes in the diagnosis and mortality risk assessment of patients with acute coronary syndrome. Indian Heart J. 2018; 70 (3): 353-359. doi: 10.1016/j.ihj.2017.09.009.

[22] Adefurin A, Darghosian L, Okafor C, et al. Alpha A adrenergic receptor genetic variation contributes to hyperglycemia after myocardial infarction. Int J Cardiol. 2016; 215: 482-486. doi: 10.1016/j.ijcard.2016.04.079.

[23] Karetnikova V, Gruzdeva O, Uchasova E et al. Glucose levels as a prognostic marker in patients with ST-segment elevation myocardial infarction: a case-control study. BMC Endocr Disord 2016; 16: 31 doi.org/10.1186/s12902-016-0108-8.

[24] Li M, Chen G, Feng $\mathrm{Y}$ and He X. Stress Induced Hyperglycemia in the Context of Acute Coronary Syndrome: Definitions, Interventions, and Underlying Mechanisms. Front Cardiovasc. Med 2021; 8: 676-892. doi: $10.3389 /$ fcvm.2021.676892.

[25] Korah TE, Ibrahim HH, Badr EA, El Shafie MK. Serum resistin in acute myocardial infarction patients with and without diabetes mellitus. Postgrad Med J. 2011 Jul; 87 (1029): 463-7. doi: 10.1136/pgmj.2010.113571.

[26] Asterholm IW, Rutkowski JM, Fujikawa T, Cho YR, Fukuda M, Tao C, Wang ZV, Gupta RK, Elmquist JK, Scherer PE. Elevated resistin levels induce central leptin resistance and increased atherosclerotic progression in mice. Diabetologia. 2014 Jun; 57 (6): 1209-18. doi: 10.1007/s00125-014-3210-3.

[27] Park HK, Kwak MK, Kim HJ, Ahima RS: Linking resistin, inflammation, and cardiometabolic diseases. The Korean Journal of Internal Medicine 2017, 32 (2): 239- 247.

[28] Hsieh, HJ., Liu, CA., Huang, B. et al. Shear-induced endothelial mechanotransduction: the interplay between reactive oxygen species (ROS) and nitric oxide (NO) and the pathophysiological implications. J Biomed Sci 2014; 21: 3. https://doi.org/10.1186/1423-0127-21-3 
[29] Samsamshariat SZA, Sakhaei F, Salehizadeh L, Keshvari M, Asgary S. Relationship between resistin, endothelin-1, and flow-mediated dilation in patient with and without metabolic syndrome. Adv Biomed Res 2019; 8: 16.

[30] Erer HB, Sayar N, Guvenc TS, Aksaray S, Yilmaz H, Altay S, Turer A, Oz TK, Karadeniz FO, Oz D, Ekmekci A, Zencirci $\mathrm{AE}$, Eren M. Prognostic value of serum resistin levels in patients with acute myocardial infarction. Kardiol Pol. 2014; 72 (2): 181-6. doi: 10.5603/KP.a2013.0086.

[31] Acquarone E, Monacelli F, Borghi R, Nencioni A, Odetti P.
Resistin: A reappraisal, Mech of Ageing and Develo 2019; 178: 46-63 https://doi.org/10.1016/j.mad.2019.01.004.

[32] Niaz S, Latif J, Hussain S. Serum resistin: A possible link between inflammation, hypertension and coronary artery disease. Pak J Med Sci 2019; 35 (3).

[33] Laurikka A, Vuolteenaho K, Toikkanen V, Rinne T, TiinaLeppänen, MattiTarkka et al. Adipocytokine resistin correlates with oxidative stress and myocardial injury in patients undergoing cardiac surgery, Eur J of Cardio-Thor Sur 2014; 46 (4): 729-736, https://doi.org/10.1093/ejcts/ezt634. 